\title{
Low expression of Toll-like receptors in peripheral blood mononuclear cells of pediatric patients with acute lymphoblastic leukemia
}

\author{
MARÍA SÁNCHEZ-CUAXOSPA ${ }^{1}$, ALEJANDRA CONTRERAS-RAMOS ${ }^{2}$, ERANDI PÉREZ-FIGUEROA ${ }^{1}$, \\ AURORA MEDINA-SANSÓN ${ }^{3}$, ELVA JIMÉNEZ-HERNÁNDEZ ${ }^{4,5}$, JOSÉ R. TORRES-NAVA ${ }^{4}$, \\ EMILIO ROJAS-CASTILLO ${ }^{6}$ and CARMEN MALDONADO-BERNAL ${ }^{1}$ \\ ${ }^{1}$ Immunology and Proteomic Research Laboratory, ${ }^{2}$ Laboratory of Developmental Biology, \\ ${ }^{3}$ Department of Hematology and Oncology, Children's Hospital of Mexico Federico Gómez, Mexico City; \\ ${ }^{4}$ Oncology Service, Children's Hospital Moctezuma, Mexico City; ${ }^{5}$ Oncology Service, Highly Specialized Unit, \\ General Hospital National Medical Center 'La Raza', Mexican Institute of Social Security, Mexico City; \\ ${ }^{6}$ Institute of Biomedical Sciences, National Autonomous University of Mexico, Mexico City, Mexico
}

Received March 26, 2016; Accepted May 16, 2016

DOI: 10.3892/ijo.2016.3569

\begin{abstract}
Cancer is the second most common cause of death among children aged 1-14 years. Leukemia accounts for one-third of all childhood cancers, $78 \%$ of which is acute lymphoblastic leukemia (ALL). The development of cancer has been associated with malignant cells that express low levels of immunogenic molecules, which facilitates their escape from the antineoplastic immune response. It is thought that it may be possible to rescue the antineoplastic immune response through the activation of recognition receptors, such as Toll-like receptors (TLRs), which activate the innate immune system. TLRs are type I membrane glycoproteins expressed mainly in immune system cells such as monocytes, neutrophils, macrophages, dendritic cells, T, B and natural killer cells. The aim of the present study was to evaluate the expression of TLR1, TLR3, TLR4, TLR7 and TLR9 in peripheral blood mononuclear cells (PBMCs) in patients with ALL and prior to any treatment. PBMCs were obtained from 50 pediatric patients diagnosed with ALL and from 20 children attending the ophthalmology and orthopedics services. The mean fluorescence intensity was obtained by analysis of immunofluorescence. We found lower expression levels of TLR1, TLR3, TLR4, TLR7 and TLR9 in PBMCs from patients with ALL compared with those from control patients. We also observed that the PBMCs from patients with Pre-B and B ALL had lower TLR4 expression than controls and patients
\end{abstract}

Correspondence to: Dr Carmen Maldonado-Bernal, Immunology and Proteomic Research Laboratory, Children's Hospital of Mexico Federico Gómez, 06720 Mexico City, Mexico

E-mail: cmaldobe@yahoo.com

Key words: acute lymphoblastic leukemia, Toll-like receptors, peripheral blood mononuclear cells, immunophenotype, innate immune system with Pro-B, Pre-B, B and T ALL had lower TLR7 expression than controls. The present study is the first to demonstrate reduced expression of TLRs in PBMCs from pediatric patients with ALL. This finding is of great relevance and may partly explain the reduction in the antineoplastic immune response in patients with ALL.

\section{Introduction}

Cancer is the second most common cause of death among children aged 1-14 years. Leukemia accounts for one-third of all cancers diagnosed in children, $78 \%$ of which is acute lymphoblastic leukemia (ALL) (1) and $10 \%$ acute myeloid leukemia (2). ALL is characterized by the uncontrolled production within the bone marrow of hematopoietic precursor cells of the lymphoid or myeloid series. Nearly $85 \%$ of ALL cases have a B-cell immunophenotype precursor and approximately $15 \%$ show a T-cell phenotype (3).

The development of cancer has been associated with malignant-like cells that express low levels of immunogenic surface molecules and are poor T-cell stimulators, which facilitates their escape from cellular antineoplastic immune responses (4). The immune system protects against invading pathogens and transformed cells, including cancer cells; the activation of the innate immune system via pattern recognition receptors such as Toll-like receptors (TLRs) is well established. TLRs are type I membrane glycoproteins with an extracellular domain containing leucine-rich repeats, which is required for the recognition of pathogen-associated molecular patterns and damage-associated molecular patterns. TLRs also have a cytoplasmic Toll/interleukin-1 receptor (TIR) domain, required for downstream signaling (5). The TLR family is composed of 12 and 10 functional receptors in mice and humans, respectively. Human TLRs are mainly expressed in immune-related cells, such as monocytes, neutrophils, macrophages, dendritic cells (DC), T, B and natural killer (NK) cells (6). The expression 
Table I. Demographic and clinical data of patients with acute lymphoblastic leukemia (ALL) and controls.

\begin{tabular}{lcc}
\hline Characteristics & ALL (n=50) & Control (n=20) \\
\hline Median age & 7 (range, 3 months & 8 (range, \\
& to 15 years) & $4-15$ years) \\
Gender & 23 F/27 M & 9 F/11 M \\
ALL immunophenotype & Pro-B 6 & \\
& Pre-B 23 & \\
& B 13 & \\
& T 8 & \\
BM blast infiltration (\%) & $70-99$ & \\
Risk stratification & SR 7 & \\
& HR 43
\end{tabular}

BM, bone marrow; M, male; F, female; Pro-B, B-cell early precursor acute lymphoblastic leukemia; B, B-cell precursor acute lymphoblastic leukemia; T, T-cell acute lymphoblastic leukemia; HR, high risk; SR, standard risk.

or upregulation of TLRs has been demonstrated in solid tumors and tumor cell lines, but their expression or role in the pathogenesis and development of acute leukemia in children remains unclear (7).

The aim of the present study was to evaluate the expression of TLR1, TLR3, TLR4, TLR7 and TLR9 in peripheral blood mononuclear cells (PBMCs) from children with acute lymphoblastic leukemia.

\section{Materials and methods}

PBMCs were obtained from 50 pediatric patients diagnosed with acute lymphoblastic leukemia (ALL) prior to any treatment. As controls, PBMC samples were obtained from 20 children attending the ophthalmology and orthopedics services. All procedures were approved by the Research, Ethics and Biosafety Committee of the Hospital Infantil de México Federico Gómez (Registry HIM/2011/022), following the International Guidelines for Biomedical Research Involving Human Beings (CIOMS-WHO 1993), and the Ethical Principles for Medical Research Involving Human Subjects of the World Medical Association and by the National Committee of Scientific Research. Samples of peripheral blood were collected after informed consent from the parents. The demographic and clinical data of the included patients are summarized in Table I.

Isolation of PBMCs. PBMCs were obtained from peripheral blood collected according to the international and institutional guidelines from pediatric patients diagnosed with ALL prior to any treatment or transfusion; cells were isolated by density gradient with Lymphoprep ${ }^{\mathrm{TM}}$ (Axis-Shield, Oslo, Norway) according to the manufacturer's instructions.

Immunofluorescence analysis. PBMCs $\left(1 \times 10^{5}\right)$ from patients with ALL were dropped onto clean glass coverslips, incubated for $15 \mathrm{~min}$ at room temperature in PBS containing 4\% parafor-
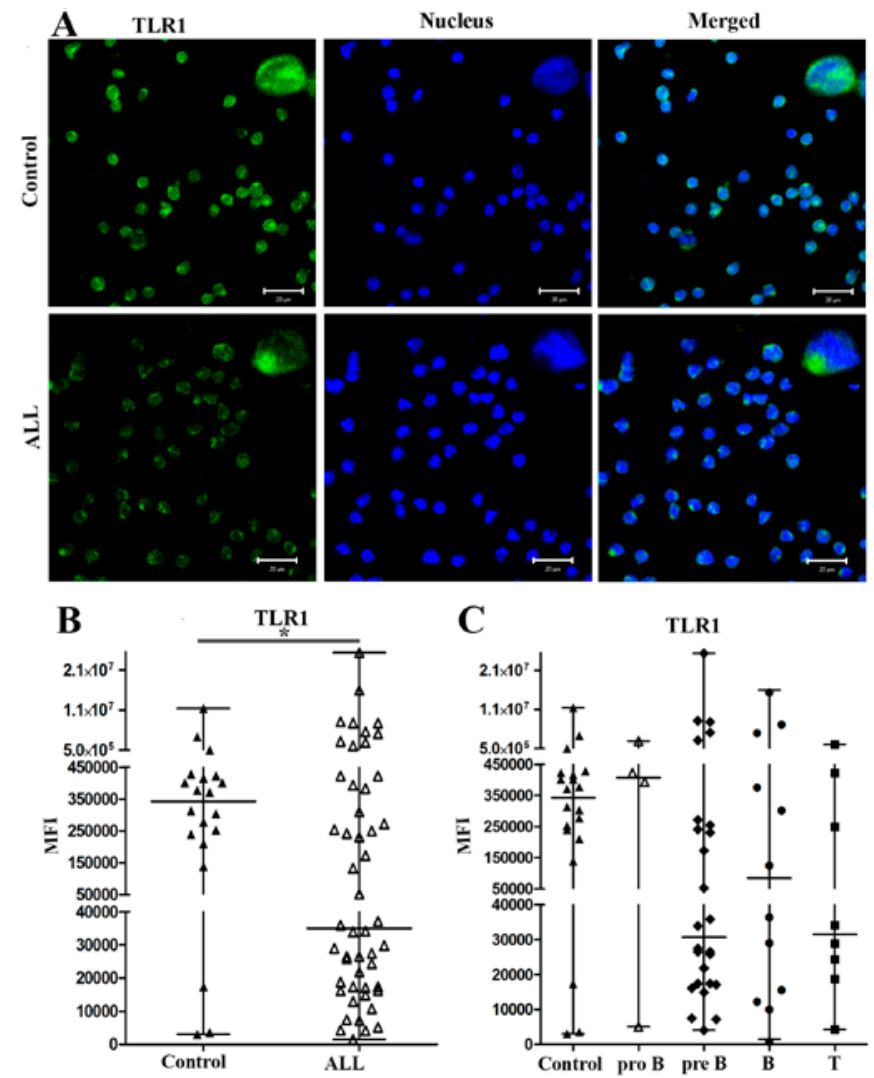

Figure 1. (A) Expression of TLR1 in peripheral blood mononuclear cells of control and ALL pediatric patients. TLR1. The expression of TLR1 detected with anti-TLR1-FITC is represented in green. Nucleus. The DRAQ7-stained nucleus is blue. Merged TLR1 and nucleus (magnification, x40). Inset detail shows expression of TLR1. (B) Quantification of the mean fluorescence intensity (MFI) for TLR1. The median MFIs of 50 patients with ALL and 20 control patients were 35,017 and 342,460 , respectively ( $\mathrm{P}<0.0349)$. The P-values were calculated using the Mann-Whitney U test. (C) The median MFI for TLR1 did not differ significantly between patients with pro-B, pre-B, $\mathrm{B}$ and T ALL. The P-values were calculated using the Kruskal-Wallis test.

maldehyde, and then washed in $0.1 \%$ PBS/Tween-20. The cells were blocked with Power Block Universal Blocking reagent (BioGenex, San Ramón, CA, USA) in a humidified chamber for $10 \mathrm{~min}$. The fixed cells were incubated with primary antibodies (goat polyclonal IgG anti-TLR1, anti-TLR3, antiTLR4, anti-TLR7 or anti-TLR9; Santa Cruz Biotechnology, Heidelberg, Germany) at room temperature for $1 \mathrm{~h}$. After two washes with $0.1 \%$ PBS/Tween-20, cells were incubated with secondary antibodies (FITC-coupled goat anti-rabbit $\operatorname{IgG}$ $(\mathrm{H}+\mathrm{L})$; Jackson ImmunoResearch, West Grove, PA, USA) for $40 \mathrm{~min}$ at room temperature and then washed twice with $0.1 \%$ PBS/Tween-20. Some glass coverslips with PBMCs were also incubated with $50 \mathrm{nM}$ of LysoTracker ${ }^{\circledR}$ (Molecular Probes Corp., CA, USA) for $30 \mathrm{~min}$ at room temperature and then washed twice with $0.1 \%$ PBS/Tween-20. Nuclei were stained for $20 \mathrm{~min}$ at room temperature with DRAQ7 (Abcam, Cambridge, MA, USA). Finally, cells were washed twice in PBS/Tween-20. The coverslips were mounted on glass slides using Vectashield mounting medium (Vector Laboratories, Burlingame, CA, USA) and dried overnight in the dark.

Confocal microscopy analysis. Cells were visualized using an Axiovert 100M LSM5 confocal microscope (Carl Zeiss, 

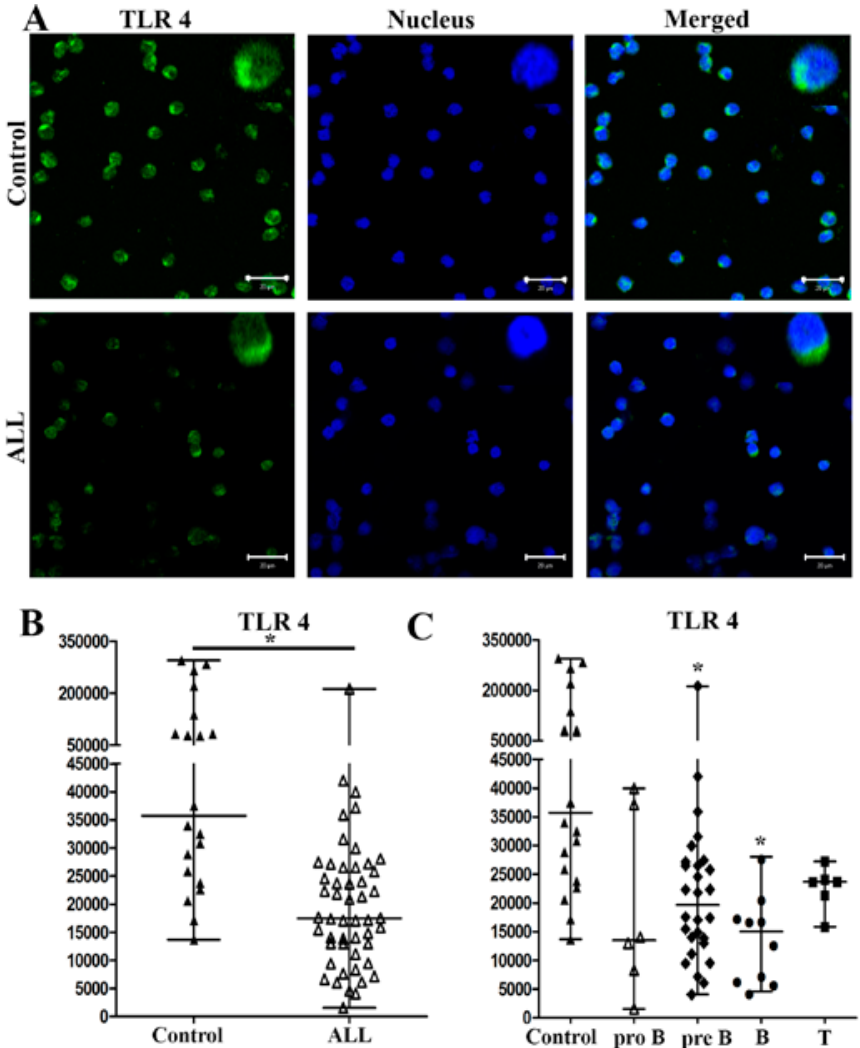

Figure 2. (A) Expression of TLR4 in peripheral blood mononuclear cells of control and ALL pediatric patients. TLR4. The expression of TLR4 detected with anti-TLR4-FITC is represented in green. Nucleus. The DRAQ7-stained nucleus is blue. Merged TLR4 and nucleus (magnification, x40). Inset detail shows the expression of TLR4. (B) Quantification of the MFI for TLR4. The medians of the MFI of 50 patients with ALL and 20 control patients were $17,478$ and 35,745 , respectively ( $\mathrm{P}<0.0001)$. The P-values were calculated using the Mann-Whitney U test. (C) The median MFI for TLR4 in patients with pro-B and B ALL was significantly different to that of the control group $\left({ }^{*} \mathrm{P}<0.05\right)$. The $\mathrm{P}$-values were calculated using the Kruskal-Wallis test.

Jena, Germany); with a x63 oil objective. Briefly, 10 micrographs were captured at $\mathrm{x} 40$ using a zoom of $1.5,488$ and $543 \mathrm{~nm}$ lasers, and BP 505-550 nm and LP 650-nm filters. In each experiment, images from different samples were taken consecutively using identical settings. Images were analyzed using ImageJ software. The results were expressed as medians of the media of the fluorescence intensity (MFI).

Statistical analysis. Prism software (version 5.01; Graphpad Software Inc., La Jolla, CA, USA) was used for statistical analysis. The comparison between groups was performed by comparing medians with the Mann-Whitney U test. The differences between phenotypes were analyzed using the Kruskal-Wallis test. P-values $<0.05$ were considered significant for both tests.

\section{Results}

Expression of TLR1 and TLR4. The patients with ALL comprised 23 girls and 27 boys; the control children were 9 girls and 11 boys. The average age of the patients with ALL was $7 \pm 4$ years (range, 3 months to 15 years), and $8 \pm 4$ years for the controls (range, 4-15 years). The expression of TLRs
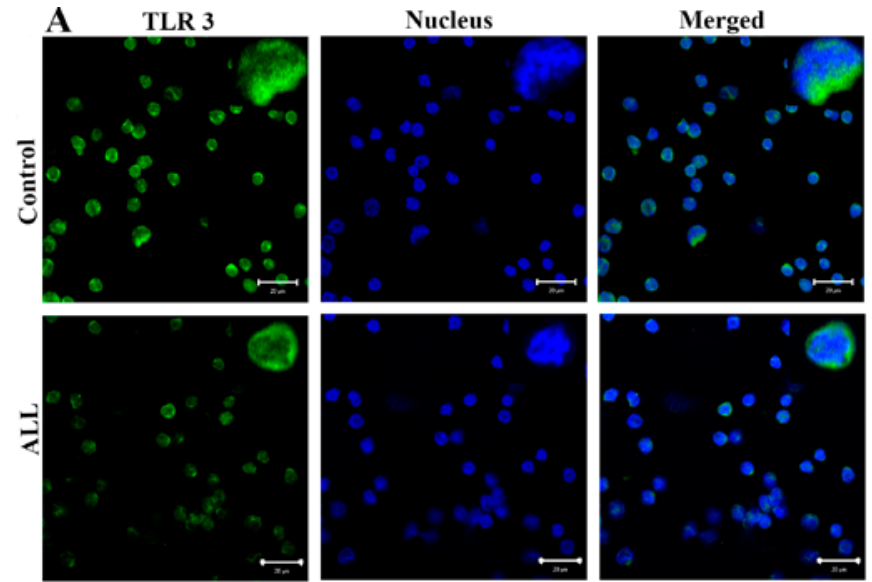

B

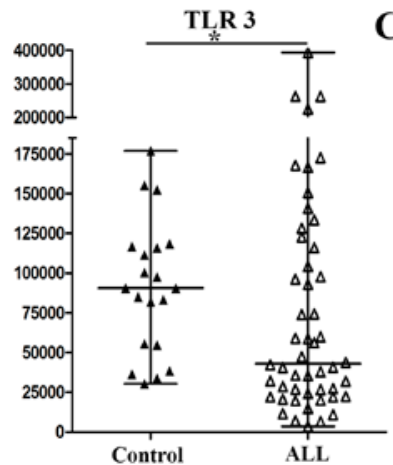

C

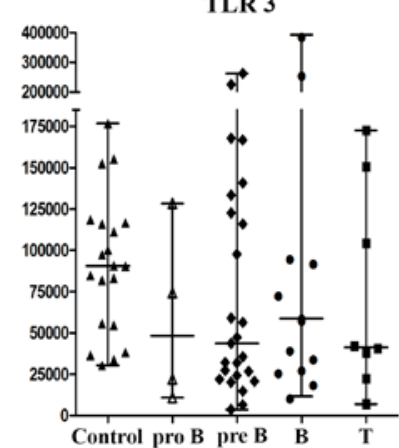

Figure 3. (A) Expression of TLR3 in peripheral blood mononuclear cells of control and ALL pediatric patients. TLR3. The expression of TLR3 detected with anti-TLR3-FITC is represented in green. Nucleus. The DRAQ7-stained nucleus is blue. Merged TLR3 and nucleus (magnification, x40). Inset detail shows expression of TLR3. (B) Quantification of the MFI for TLR3. The median MFIs of 50 patients with ALL and 20 control patients were 42,900 and 90,551, respectively ( $\left.{ }^{*} \mathrm{P}<0.0252\right)$. The $\mathrm{P}$-values were calculated using the Mann-Whitney U test. (C) The median MFI for TLR3 did not differ significantly between patients with pro-B, pre-B, B and T ALL. The P-values were calculated using the Kruskal-Wallis test.

in PBMCs from all patients and controls was observed by immunofluorescence. Expression of TLR1 for one representative control and for one representative patient with ALL is shown in Fig. 1A; TLR4 expression of one representative control and for one representative patient with ALL is shown in Fig. 2A. In patients with ALL, TLR1 and TLR4 expression was lower than in control patients. The median values of the mean fluorescence intensity (MFI) for TLR1 in cells from 50 patients with ALL and 20 controls were 35,017 and 342,460, respectively $(\mathrm{P}<0.0349)$ (Fig. 1B). The medians of the MFI for TLR4 in cells from patients with ALL and controls were 17,478 and 35,745, respectively ( $\mathrm{P}<0.0001)$ (Fig. 2B).

When we analyzed the expression levels of TLR1 and TLR4 in PBMCs from patients with different subtypes of ALL (pro-B, pre-B, B and T), we found that the median values of the MFI for TLR1 in pro-B, pre-B, B and T ALL were 407,631, 30,676, 84,650 and 31,531, respectively, and 342,460 for controls (Fig. 1C). The medians of the MFI for TLR4 from patients with pro-B, pre-B, B and T ALL were $13,520,19,696,15,067$ and 23,680 , respectively and 35,745 for controls (Fig. 2C). The differences in TLR4 expression were only significant between ALL pre-B and B subtypes compared with controls $(\mathrm{P}<0.0002)$. 

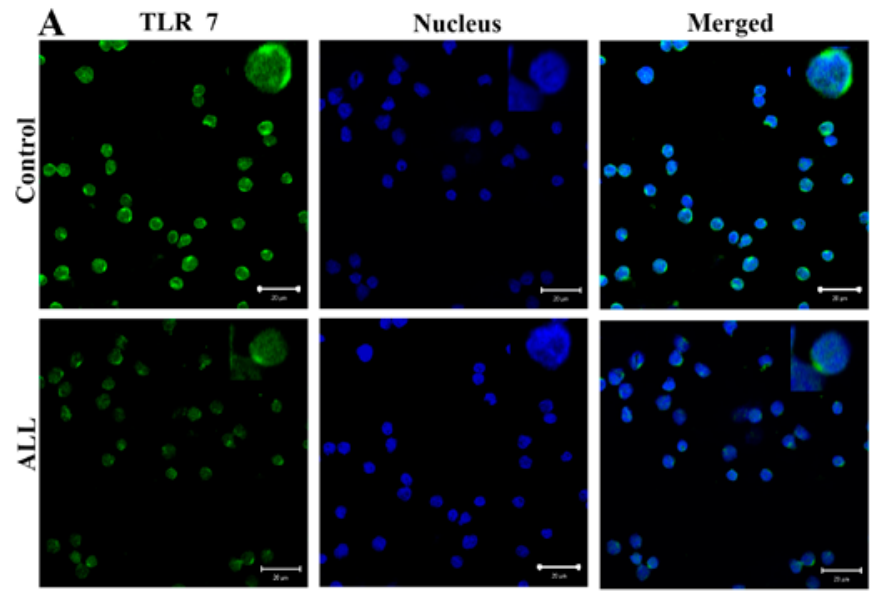

B

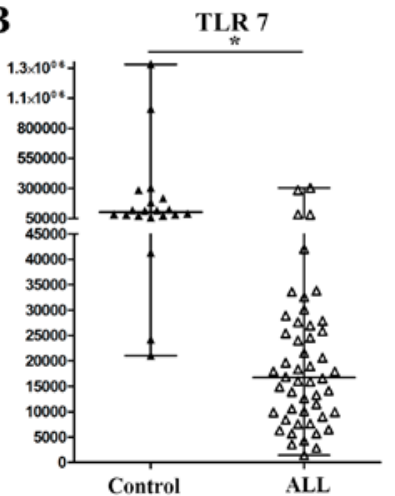

$\mathrm{C}$

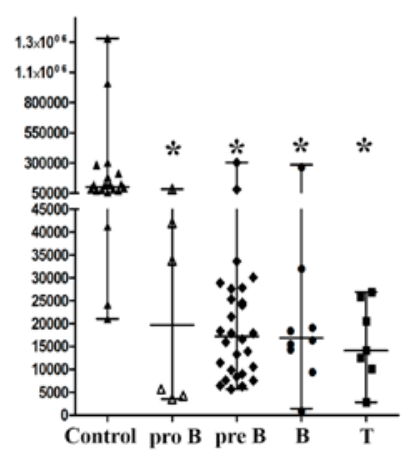

Figure 4. (A) Expression of TLR7 in peripheral blood mononuclear cells of control and ALL pediatric patients. TLR7. The expression of TLR7 detected with anti-TLR7-FITC is represented in green. Nucleus. The DRAQ7-stained nucleus is in blue. Merged TLR7 and nucleus (magnification, $x 40$ ). Inset detail shows expression of TLR7. (B) Quantification of the MFI for TLR7. The median MFIs of 50 patients with ALL and 20 control patients were 16,696 and 102,557 , respectively $\left({ }^{*} \mathrm{P}<0.0001\right)$. The $\mathrm{P}$-values were calculated using the Mann-Whitney U test. (C) The median MFI for TLR7 differed significantly between subtypes of ALL compared with controls and between groups $\left({ }^{*} \mathrm{P}<0.05\right)$. The $\mathrm{P}$-values were calculated using the Kruskal-Wallis test.
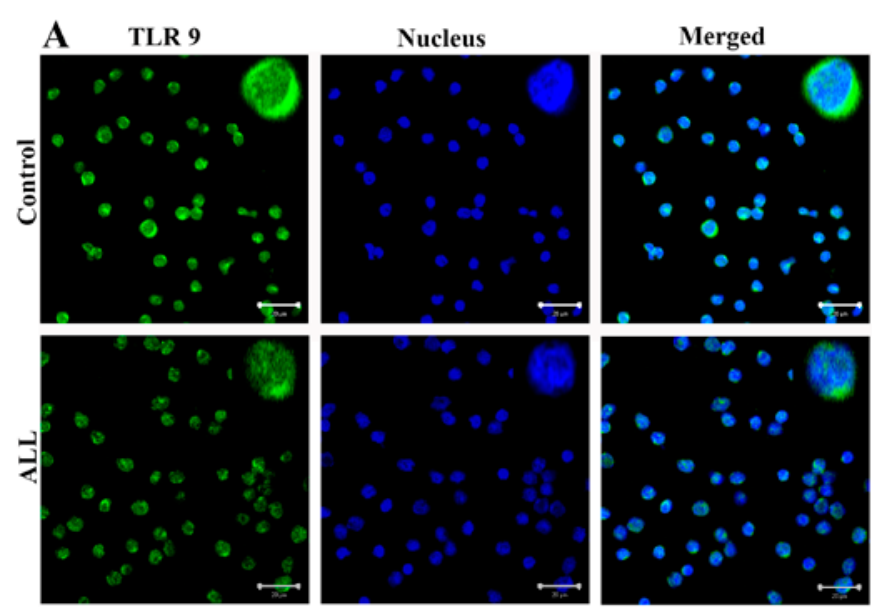

B
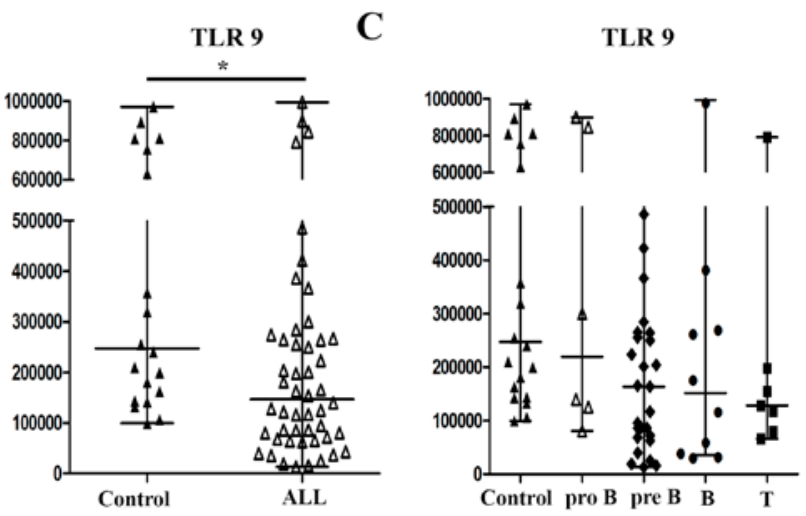

Figure 5. (A) Expression of TLR9 in peripheral blood mononuclear cells of control and ALL pediatric patients. TLR9. The expression of TLR9 detected with anti-TLR9-FITC is represented in green. Nucleus. The DRAQ7-stained nucleus is blue. Merged TLR9 and nucleus (magnification, x40). Inset detail shows expression of TLR9. (B) Quantification of the MFI for TLR9. The median MFIs of 50 patients with ALL and 20 control patients were 147,157 and 247,630, respectively ( $\left.{ }^{*} \mathrm{P}<0.0038\right)$. The P-values were calculated using the Mann-Whitney U test. (C) The median MFI for TLR9 did not differ significantly between subtypes of ALL and controls or between groups. The P-values were calculated using the Kruskal-Wallis test.
Expression of TLR3, TLR7 and TLR9. By immunofluorescence, we observed different expression levels of TLR3, TLR7 and TLR9 in PBMCs from patients with ALL and controls. In Figs. 3-5, we show the results for a representative control and of a representative patient with ALL (Figs. 3A, 4A and $5 \mathrm{~A}$, respectively). The medians of the MFI for TLR3 on cells from patients with ALL and controls were 42,900 and 90,551, respectively $(\mathrm{P}<0.0252)$ (Fig. 3B). The medians of the MFI for TLR7 on cells from patients with ALL and controls were $16,696$ and 102,557, respectively ( $\mathrm{P}<0.0001)$ (Fig. 4B). Finally, the medians of the MFI for TLR9 on cells from patients with ALL and controls were 147,157 and 247,630, respectively $(\mathrm{P}<0.0038)$ (Fig. 5B).

When we analyzed the expression levels of TLR3, TLR7 and TLR9 in PBMCs from patients with pro-B, pre-B, B and T ALL, the medians of the MFI for TLR3 from patients with pro-B, pre-B, B and T ALL were 48,160, 43,674, 58,708 and 41,323, respectively, and 90,551 for controls (Fig. 3C). The medians of the MFI for TLR7 from pro-B, pre-B, B and T ALL were 19,732, 17,213, 16,841 and 14,127, respectively, and 102,557 for controls (Fig. 4C). The medians of the MFI for TLR9 from patients with pro-B, pre-B, B and T ALL were $219,857,163,306,151,555$ and 128,391 , respectively, and 247,630 for controls (Fig. 5C). The differences in TLR7 expression between ALL subtypes pro-B, pre-B, B and T compared with controls were all significant $(\mathrm{P}<0.0001)$.

When we used LysoTracker, a fluorescent probe for lysosomes, we did not find TLR1 and TLR4 in lysosomes of PBMCs from patients with ALL or controls. However, TLR3, TLR7 and TLR9 were present in lysosomes of PBMCs from both patients with ALL and controls (data no shown).

\section{Discussion}

In the present study, we report reduced expression of TLR1, TLR3, TLR4, TLR7 and TLR9 in PBMCs from patients with ALL compared with controls. We observed substantial heterogeneity in the level and type of TLR expression in the patients with ALL. Our results also showed that Pro-B, Pre-B, B and T ALL phenotypes presented the lowest levels of TLR7 expression while Pre-B and B ALL phenotypes presented the lowest levels of TLR4 expression. There were no significant differences for the other TLRs and leukemia phenotypes, possibly because there was a broad distribution of expression and only 
a small number of patients with each ALL phenotype. We observed TLR3, TLR7 and TLR9 but not TLR1 and TLR4 in lysosomes of PBMCs from ALL patients and controls.

Studies have analyzed the expression of TLRs in tonsil tissues (8), autoimmune diseases (9-11), in solid cancers (12), hematological cancers such as multiple myeloma and chronic myeloid leukemia (CML) $(13,14)$, and in murine models $(15,16)$, cell lines (17) and PBMCs from CML patients (18). However, this is the first study to analyze the expression level of TLRs in PBMCs from pediatric patients with ALL.

One study demonstrated TLR1 and TLR6 mRNA expression in all cell types of PBMCs in adult humans by quantitative RT-PCR; this group also detected high expression of TLR7 mRNA and TLR9 mRNA in DCs and marked levels of TLR2 mRNA in monocytes (19). Naïve B cells expressed low to undetectable levels of TLR1, TLR2 and TLR4, while memory B cells expressed constitutively high levels of TLR6, TLR7, TLR10 and especially TLR9 (18). Another study found high expression levels of TLR1, TLR2, TLR7, TLR9 and TLR10 in germinal center and memory B cells in tonsils, while TLR9 levels were lower in circulating blood B cells (8). Other studies have also noted that specific subsets of human peripheral $\mathrm{T}$ cells might selectively express different TLRs (TLR1-5, 7 and 9 mRNA), although at greatly varying levels (19). Specifically, regulatory $\mathrm{T}$ cells (Treg), but not naïve T cells, expressed TLR8 (20); Treg also expressed TLR4 and TLR5 mRNA $(21,22)$.

In the present study, we report lower expression levels of all TLRs studied in PBMCs from ALL patients compared with that in the controls; the lowest expression of TLR4 and TLR7 was observed in children with Pro-B and B ALL subtypes. TLR expression may differ depending on stimulus, environment, subset, and cell type and probably age group. The TLR expression profile appears to be influenced by the location of the cells; in this case it is of interest because it occurs in a pathological condition and because all studied TLRs were expressed at lower levels than in the controls.

As a way to study TLR expression in hematological cancer, Bourke et al (17) used a panel of cell lines derived from different leukemia and lymphoma cell types to demonstrate expression of TLR9 and/or TLR10, although pre-B-cell lines were negative. Other studies reported highly variable expression of mRNA for TLRs1-7 in B-cell precursor ALL cell lines (23). Expression of TLRs in PBMCs from patients with B-cell chronic lymphocytic leukemia (B-CLL) was restricted to TLR7, 8 and 9 (18). TLR7 is expressed by plasmacytoid DCs and memory B cells (24). Studies in patients with CLL showed TLR1, TLR2, TLR6 and TLR10 expression (25). Patients with acute myeloid leukemia (AML) expressed TLR2, 4 and 9 in monocyte-derived DCs; healthy individuals strongly expressed TLR2 and TLR4, and TLR9 was expressed at a lower level in both groups (26-28). Nevertheless, pre-B ALL cells expressed TLR1, 7 and 9, and low levels of TLR3, 4 and 5. This may be a consequence of malignant differentiation or just a reflection of the normal B-cell precursor phenotype (5).

We report low expression of TLR1, 3, 4, 7 and 9 in PBMCs from pediatric patients with ALL compared with those from control children. These results show that healthy children and those with neoplasia express different levels of TLRs. It is probable that the age of these patients adds to these differences, because age plays an important role in linking innate and adaptive immune responses in normal and pathologic conditions. A high level of heterogeneity exists between different reports studying patients and cell lines; in some cases, this may be associated with disease activity.

Most studies of TLR4 expression levels, including our own, have reported it as decreased in patients with leukemia, which might be related to the development or presence of a leukemic clone of cells in lymphocytic leukemia and myeloid leukemia $(29,30)$. Thus, further studies are required to determine whether this decreased TLR4 expression contributes to the pathogenesis of leukemic clone development through an associated depressed immune surveillance (31), or whether patients have an increased risk of disease progression and poor prognosis with the development of autoimmune complications (29).

The decreased TLR4 expression in pre-B and B ALL subtypes observed in this study might therefore be a reflection of an impaired host response toward the malignant clonal populations. In the present study, levels of TLR4 expression in both subsets of ALL were lower than in controls; therefore, depressed resistance to the challenge of leukemic transformation might be associated with a lack of sufficient host TLR4 (30). However, normal B cells also responded to TLR7 activation by increasing costimulatory molecule expression, cytokine production and by becoming more sensitive to killing by cytotoxic effectors (25). These findings suggest a potential role for TLR4 and TLR7 in disease progression and in inhibition of effective immunotherapy.

Physiologically, various diseases may alter the immune regulatory function of TLRs; stimulation with different ligands could be crucial for the antitumor immune response. Bekeredjian-Ding et al (31) showed that in peripheral blood B cells of healthy donors, type I interferon (IFN), induced during infections, triggered TLR7 expression and polyclonal B-cell expansion and also B-cell differentiation toward plasma cells. In addition, Reid et al (32) showed that the impact of $\mathrm{CpG}$ stimulation on precursor B ALL cell lines and B ALL bone marrow biopsy samples from pediatric patients was characterized by increased CD40 expression but only small changes in CD86 levels and no induction of CD80 expression. CpG stimulation of ALL blasts produced increased levels of interleukin-6 (IL-6), IL-8, IL-10 and IFN- $\gamma$, but no detectable IL-12p70, and reduced secretion of IL-5. Agonists of TLR2, TLR4, TLR7 and TLR9 led to detectable changes in costimulatory molecule expression in ALL; only TLR2 and TLR9 stimulation influenced T-cell responses $(24,30)$. Unlike the findings in other B-cell leukemia types, no change in proliferation was observed for pre-B ALL cells in the presence of ligands for TLR2, TLR7 and TLR9 (32).

TLR expression is present in both normal immune cells and in malignant cells, but with distinctive patterns (6), so the expression of TLRs in pre-B leukemic blasts compared with normal $\mathrm{B}$ cells may be the result of malignant transformation or simply a reflection of the underlying phenotype of the precursor cells. TLR agonists might serve to strengthen effectively the response of the immune system in leukemia.

The expression of TLRs has been demonstrated in some tumors and tumor cell lines, but the role of TLRs in pathogenesis and/or development of acute leukemia remains unclear. 
The expression of TLRs in hematological cancer has been demonstrated in murine models, cell lines, bone marrow, B and $\mathrm{T}$ cells of peripheral blood samples from patients with non-Hodgkin lymphoma, multiple myeloma, AML, CML and in healthy volunteers. However, the expression of TLRs in PBMCs from pediatric patients with ALL has not been reported previously; this is the first study reporting the expression of TLRs 1, 3, 4, 7 and 9 in PBMCs from children with ALL.

The present study provides information on the expression of TLRs in cells of the immune system that are crucial for the antitumor immune response. Although the possible role of TLR in the pathogenesis of these diseases is unclear, the low level of expression of TLRs in patients with ALL may partially explain the deficiency that exists in the antitumor immune response of these patients. The observed variations could also explain the differences in the responses of the patients to autoimmune diseases, infection, and neoplastic responses, including active disease. Although our results show low expression of some TLRs, further studies are necessary to address the expression of TLRs in different mononuclear cell types. Variation between cell populations must be analyzed to determine whether the differences found between patients with ALL and controls are related to different levels of expression in different types of mononuclear cells and/or subtypes of ALL.

\section{Acknowledgements}

The present study was supported by the Hospital Infantil de México Federico Gómez, grant HIM/2011/022,HIM/2012/024, 06720 Mexico City, Mexico. María A. Sánchez-Cuaxospa acknowledges the scholarship provided by CONACyT. This study constitutes a partial fulfillment of the Graduate Program of Doctorado en Ciencias Biomédicas of the Universidad Nacional Autonóma de México (UNAM).

\section{References}

1. Siegel R, Naishadham D and Jemal A: Cancer statistics, 2012. CA Cancer J Clin 62: 10-29, 2012.

2. Stock W and Pui CH: Chapter 17. Acute lymphoblastic leukemia and lymphoblastic lymphoma. ASH-SAP, pp489-510, 2010.

3. Hossain MJ, Xie L and Caywood EH: Prognostic factors of childhood and adolescent acute myeloid leukemia (AML) survival: Evidence from four decades of US population data. Cancer Epidemiol 39: 720-726, 2015.

4. Rakoff-Nahoum S and Medzhitov R: Toll-like receptors and cancer. Nat Rev Cancer 9: 57-63, 2009.

5. Chiron D, Bekeredjian-Ding I, Pellat-Deceunynck C, Bataille R and Jego G: Toll-like receptors: Lessons to learn from normal and malignant human B cells. Blood 112: 2205-2213, 2008.

6. Harsini S, Beigy M, Akhavan-Sabbagh M and Rezaei N: Toll-like receptors in lymphoid malignancies: Double-edged sword. Crit Rev Oncol Hematol 89: 262-283, 2014.

7. Fabricius D, Breckerbohm L, Vollmer A, Queudeville M, Eckhoff SM, Fulda S, Strauss G, Debatin KM, Jahrsdörfer B and Meyer LH: Acute lymphoblastic leukemia cells treated with $\mathrm{CpG}$ oligodeoxynucleotides, IL-4 and CD40 ligand facilitate enhanced anti-leukemic CTL responses. Leukemia 25: 1111-1121, 2011.

8. Månsson A, Adner M, Höckerfelt U and Cardell LO: A distinct Toll-like receptor repertoire in human tonsillar B cells, directly activated by PamCSK, R-837 and CpG-2006 stimulation. Immunology 118: 539-548, 2006.

9. Marshak-Rothstein A: Toll-like receptors in systemic autoimmune disease. Nat Rev Immunol 6: 823-835, 2006.
10. Krieg AM and Vollmer J: Toll-like receptors 7, 8, and 9: Linking innate immunity to autoimmunity. Immunol Rev 220: 251-269, 2007.

11. Klonowska-Szymczyk A, Wolska A, Robak T, Cebula-Obrzut B, Smolewski P and Robak E: Expression of toll-like receptors 3, 7, and 9 in peripheral blood mononuclear cells from patients with systemic lupus erythematosus. Mediators Inflamm 2014: 381418, 2014.

12. Green TL, Santos MF, Ejaeidi AA, Craft BS, Lewis RE and Cruse JM: Toll-like receptor (TLR) expression of immune system cells from metastatic breast cancer patients with circulating tumor cells. Exp Mol Pathol 97: 44-48, 2014.

13. Xu Y, Zhao Y, Huang H, Chen G, Wu X, Wang Y, Chang W, Zhu Z, Feng Y and Wu D: Expression and function of toll-like receptors in multiple myeloma patients: Toll-like receptor ligands promote multiple myeloma cell growth and survival via activation of nuclear factor-kappaB. Br J Haematol 150: 543-553, 2010.

14. Bernasconi NL, Onai N and Lanzavecchia A: A role for Toll-like receptors in acquired immunity: Up-regulation of TLR9 by BCR triggering in naive $\mathrm{B}$ cells and constitutive expression in memory B cells. Blood 101: 4500-4504, 2003.

15. Genestier L, Taillardet M, Mondiere P, Gheit H, Bella C and Defrance T: TLR agonists selectively promote terminal plasma cell differentiation of B cell subsets specialized in thymusindependent responses. J Immunol 178: 7779-7786, 2007.

16. Gururajan M, Jacob J and Pulendran B: Toll-like receptor expression and responsiveness of distinct murine splenic and mucosal B-cell subsets. PLoS One 2: e863, 2007.

17. Bourke E, Bosisio D, Golay J, Polentarutti N and Mantovani A: The toll-like receptor repertoire of human B lymphocytes: Inducible and selective expression of TLR9 and TLR10 in normal and transformed cells. Blood 102: 956-963, 2003.

18. Spaner DE, Miller RL, Mena J, Grossman L, Sorrenti V and Shi Y: Regression of lymphomatous skin deposits in a chronic lymphocytic leukemia patient treated with the Toll-like receptor-7/8 agonist, imiquimod. Leuk Lymphoma 46: 935-939, 2005.

19. Hornung V, Rothenfusser S, Britsch S, Krug A, Jahrsdörfer B, Giese T, Endres $S$ and Hartmann G: Quantitative expression of toll-like receptor 1-10 mRNA in cellular subsets of human peripheral blood mononuclear cells and sensitivity to $\mathrm{CpG}$ oligodeoxynucleotides. J Immunol 168: 4531-4537, 2002.

20. Peng G, Guo Z, Kiniwa Y, Voo KS, Peng W, Fu T, Wang DY, Li Y, Wang HY and Wang RF: Toll-like receptor 8-mediated reversal of $\mathrm{CD}^{+}$regulatory $\mathrm{T}$ cell function. Science 309: 1380-1384, 2005.

21. Crellin NK, Garcia RV, Hadisfar O, Allan SE, Steiner TS and Levings MK: Human CD4 ${ }^{+} \mathrm{T}$ cells express TLR5 and its ligand flagellin enhances the suppressive capacity and expression of FOXP3 in $\mathrm{CD} 4{ }^{+} \mathrm{CD} 25^{+} \mathrm{T}$ regulatory cells. J Immunol 175: 8051-8059, 2005.

22. Caramalho I, Lopes-Carvalho T, Ostler D, Zelenay S, Haury M and Demengeot J: Regulatory $\mathrm{T}$ cells selectively express toll-like receptors and are activated by lipopolysaccharide. J Exp Med 197: 403-411, 2003.

23. Corthals SL, Wynne K, She K, Shimizu H, Curman D, Garbutt K and Reid GS: Differential immune effects mediated by Toll-like receptors stimulation in precursor B-cell acute lymphoblastic leukaemia. Br J Haematol 132: 452-458, 2006.

24. Spaner DE, Shi Y, White D, Mena J, Hammond C, Tomic J, He L, Tomai MA, Miller RL, Booth J, et al: Immunomodulatory effects of Toll-like receptor-7 activation on chronic lymphocytic leukemia cells. Leukemia 20: 286-295, 2006.

25. Muzio M, Scielzo C, Bertilaccio MT, Frenquelli M, Ghia P and Caligaris-Cappio F: Expression and function of toll like receptors in chronic lymphocytic leukaemia cells. Br J Haematol 144: 507-516, 2009.

26. Schmitt A, Li L, Giannopoulos K, Greiner J, Reinhardt P, Wiesneth M and Schmitt M: Quantitative expression of Toll-like receptor- $2,-4$, and -9 in dendritic cells generated from blasts of patients with acute myeloid leukemia. Transfusion 48: 861-870, 2008.

27. Rybka J, Butrym A, Wróbel T, Jaźwiec B, Stefanko E, Dobrzyńska O, Poręba R and Kuliczkowski K: The expression of Toll-like receptors in patients with acute myeloid leukemia treated with induction chemotherapy. Leuk Res 39: 318-322, 2015. 
28. Rozková D, Novotná L, Pytlík R, Hochová I, Kozák T, Bartůnková J and Spísek R: Toll-like receptors on B-CLL cells: Expression and functional consequences of their stimulation. Int J Cancer 126: 1132-1143, 2010.

29. Barcellini W, Imperiali FG, Zaninoni A, Reda G, Consonni D, Fattizzo B, Lonati S, Nobili L, Zanella A and Cortelezzi A: Toll-like receptor 4 and 9 expression in B-chronic lymphocytic leukemia: Relationship with infections, autoimmunity and disease progression. Leuk Lymphoma 55: 1768-1773, 2014.

30. Webb RN, Cruse JM and Lewis RE: Decreased TLR4 gene expression in leukemic leukocyte populations. Exp Mol Pathol 87: $117-126,2009$
31. Bekeredjian-Ding IB, Wagner M, Hornung V, Giese T, Schnurr M, Endres S and Hartmann G: Plasmacytoid dendritic cells control TLR7 sensitivity of naive B cells via type I IFN. J Immunol 174: 4043-4050, 2005.

32. Reid GS, She K, Terrett L, Food MR, Trudeau JD and Schultz KR: $\mathrm{CpG}$ stimulation of precursor B-lineage acute lymphoblastic leukemia induces a distinct change in costimulatory molecule expression and shifts allogeneic T cells toward a Th1 response. Blood 105: 3641-3647, 2005. 